\title{
Appendicitis presenting as cardiac tamponade
}

\author{
Yader Sandoval, MD, ${ }^{a}$ David M. Williams, DO, ${ }^{\mathrm{b}}$ and Steven R. Goldsmith, MD ${ }^{\mathrm{a}, \mathrm{c}}$
}

\author{
A 52-year-old man was seen with profound hypotension \\ and respiratory distress requiring emergency intubation. \\ Bedside ultrasonography in the subcostal window revealed \\ a large pericardial effusion along with a liver mass \\ (Figure 1, $A$ and $B$ ). Computed tomography confirmed

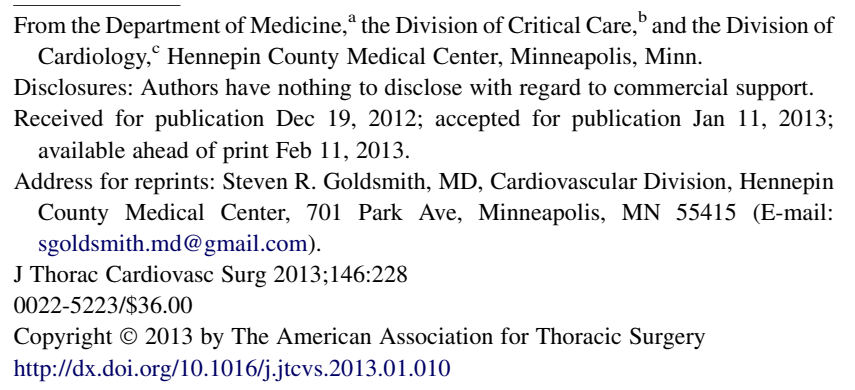

a large liver mass protruding into the pericardial sac, raising concern for fistula tracking into pericardium (Figure 1,C). The patient rapidly began to show clinical signs of pericardial tamponade. Emergency parasternal pericardiocentesis drained $450 \mathrm{~mL}$ of purulent fluid, resulting in dramatic clinical improvement. Cultures of the fluid grew Streptococcus milleri and Bacteroides fragilis. The source of the infection causing the hepatic abscess and pyopericardium was a subacute ruptured appendix that had formed an appendicealcolonic fistula, allowing bacterial transmission through the portal system, reaching the liver and, through the fistula, into the pericardium (Figure 1,D). The patient survived the episode; however, profound constrictive pericarditis developed within weeks, necessitating pericardiectomy. The patient was subsequently discharged home and is now well.
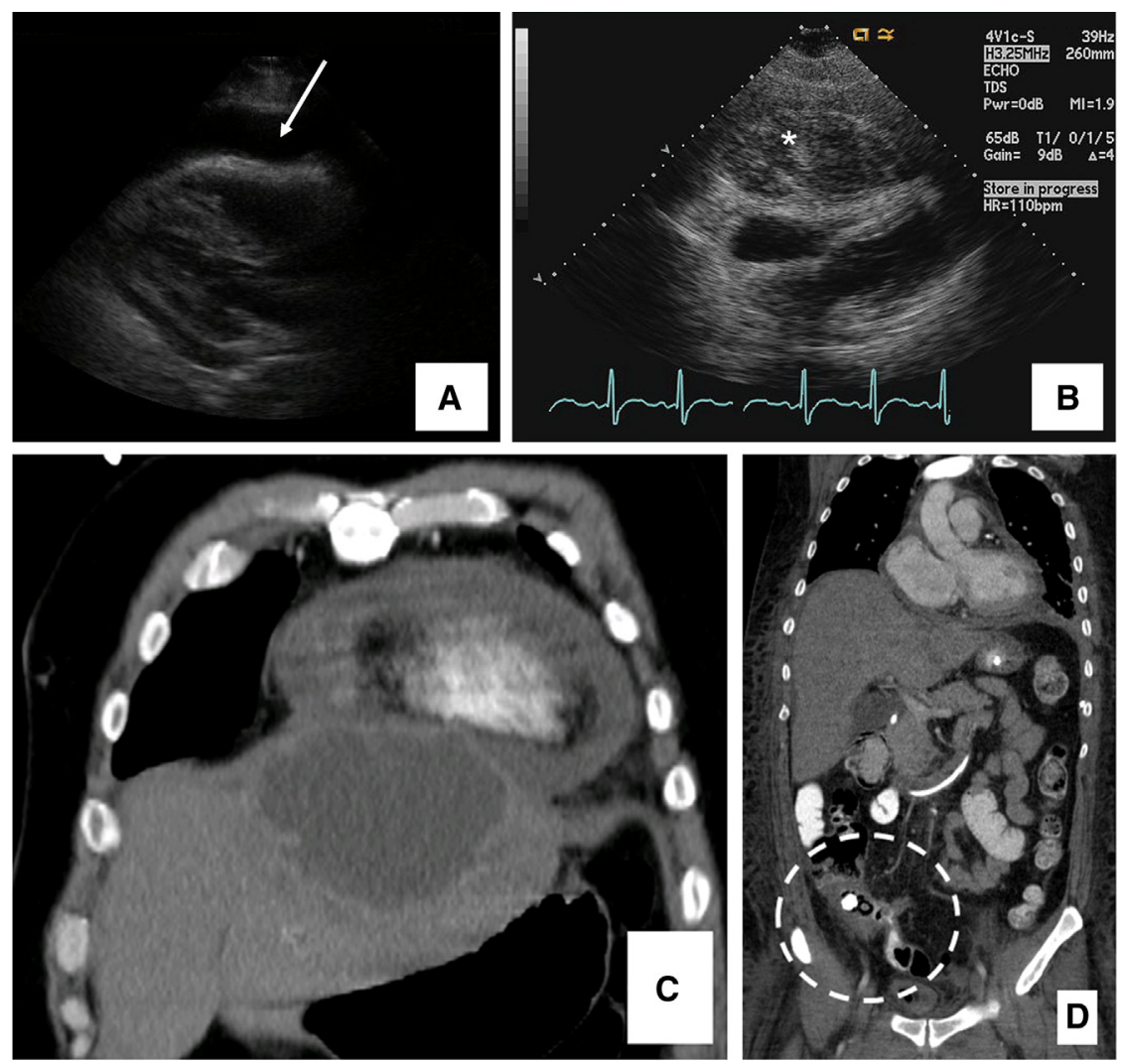

FIGURE 1. A, Transthoracic echocardiography in the parasternal long-axis view shows a large pericardial effusion (arrow). B, Transthoracic echocardiography in the subcostal view shows a liver mass (asterisk). C, Computed tomography in the coronal plane shows a large liver mass protruding into the pericardial sac. D, Computed tomography in the coronal plane shows appendiceal-colonic fistula and appendicolith (within dotted circle). 